\title{
ARTICLE OPEN \\ Bio-inspired self-folding strategy to break the trade-off between strength and ductility in carbon-nanoarchitected
} materials

\author{
Xiangzheng Jia (iD) ${ }^{1}$, Ze $\mathrm{Liu}^{1}$ and Enlai Gao ${ }^{1 *}$
}

\begin{abstract}
Graphene possesses extraordinary mechanical, electronic, and thermal properties, thus making it one of the most promising building blocks for constructing macroscopic high performance and multifunctional materials. However, the common material strength-ductility paradox also appears in the carbon-nanoarchitected materials and some of the key mechanical performance, for example, the tensile strength of graphene-based materials, are still far lower than that of graphene. Inspired by the exceptional mechanical performance of silk protein benefiting from the conformations of folded structures as well as their transitions, this work proposed a topological strategy to yield graphene-based materials with ultrahigh ductility while maintaining decent tensile strength by self-folding graphene sheets. This drastically improved mechanical performance of graphene-based materials is attributed to the exploitation of shearing, sliding, and unfolding deformation at the self-folded interface. Molecular dynamics simulations show that both modulating self-folded length and engineering interface interaction can effectively control the strength, ductility, and the ductile failure of van der Waals interfaces among the self-folded structures, where interfacial shearing, sliding, and unfolding open channels to dissipate mechanical energy. Based on the insights into the atomic-scale deformation by molecular dynamics simulations, the underlying mechanism of deformation and failure of these materials is finally discussed with a continuum mechanics-based model. Our findings bring perceptive insights into the microstructure design of strong-yet-ductile materials for load-bearing engineering applications.
\end{abstract}

npj Computational Materials (2020)6:13; https://doi.org/10.1038/s41524-020-0279-8

\section{INTRODUCTION}

Graphene has attracted considerable attention owing to its excellent thermal, ${ }^{1}$ electronic ${ }^{2}$, and mechanical ${ }^{3}$ properties. The tensile stiffness, tensile strength, and fracture strain of graphene can reach as high as $1 \mathrm{TPa}, 120 \mathrm{GPa}$ and $15 \%$, respectively, ${ }^{3}$ making it a promising candidate for constructing macroscopic materials that inherit the superior mechanical properties of this carbon nanostructure. In the past decades, considerable theoretical, computational, and experimental works have been devoted to the rational design of multi-layer graphene assemblies. On the theoretical and computational sides, studies have been conducted on the theoretical modeling and computational simulations of multi-layer graphene assemblies. Considering the in-plane tensile deformation of the building blocks, Liu et al. ${ }^{4}$ and Wei et al. ${ }^{5}$ proposed the shear-lag model for the layered/bundled assemblies of carbon nanostructures, and recently $\mathrm{He}$ et al..$^{6}$ generalized this model by introducing elastic-plastic interface. By virtue of this shear-lag model, the overall mechanical response and structural deformation of multi-layer graphene assemblies can be correlated for material analysis and design., ${ }^{4,6-8}$ Subsequently, Ruiz et al. ${ }^{9}$ developed a coarse-grained molecular dynamics (CGMD) model of multi-layer graphene assemblies. By using this CGMD model, Xia et al. ${ }^{10}$ investigated the mechanical behaviors of multi-layer graphene assemblies and found that critical length scales and strain localization govern the mechanical behaviors of these assemblies. Meanwhile, the CGMD model was also applied to design multi-layer graphene assemblies for enhanced toughness. ${ }^{11}$ Furthermore, Meng et al. ${ }^{12}$ conducted ballistic impact simulations of multi-layer graphene assemblies and demonstrated that a spalling-like failure by cylindrical projectiles deteriorates the ballistic performance of these materials. On the experimental side, recent studies used various methods, such as nanoindentation, ${ }^{13}$ in situ tensile test, ${ }^{14}$ and projectile penetration ${ }^{15}$ to characterize the mechanical behaviors of multi-layer graphene assemblies. Combining nanomechanical experiments and CGMD simulations, Wei et al. ${ }^{13}$ found that both the tensile strength and Young's modulus of multi-layer graphene assemblies decrease with increasing thickness and elucidated the mechanisms therein. Lee et al. ${ }^{15}$ studied the dynamic mechanical behaviors of multi-layer graphene assemblies by supersonic projectile penetration and demonstrated that their energy dissipation capacity can suppress conventional protective materials. Recently, Li et al. ${ }^{14}$ characterized the in-plane fracture behaviors of multi-layer graphene assemblies under tensile loading by using in situ electron microscopy and found that the fracture strength of multi-layer graphene assemblies decreases as the thickness increases. Additionally noted that considerable defects in the building blocks of graphene sheets have been characterized by experiments, ${ }^{16}$ and the load transfer through the van der Waals interfaces of graphene assemblies is weak, ${ }^{8}$ which limits the mechanical performance of graphene-based materials. To enhance the mechanical properties of graphene-based materials, two main strategies including improving the quality of the building blocks and modifying the interface interaction between building blocks were developed. ${ }^{17}$ The first strategy includes developing and modulating the intrinsic chemical structure of graphene building blocks. ${ }^{18-31}$ For example, Zhang et al. ${ }^{30}$ prepared graphene films with a tensile strength of $453 \mathrm{MPa}$ by

\footnotetext{
1Department of Engineering Mechanics, School of Civil Engineering, Wuhan University, Wuhan, Hubei 430072, China. *email: enlaigao@whu.edu.cn
} 


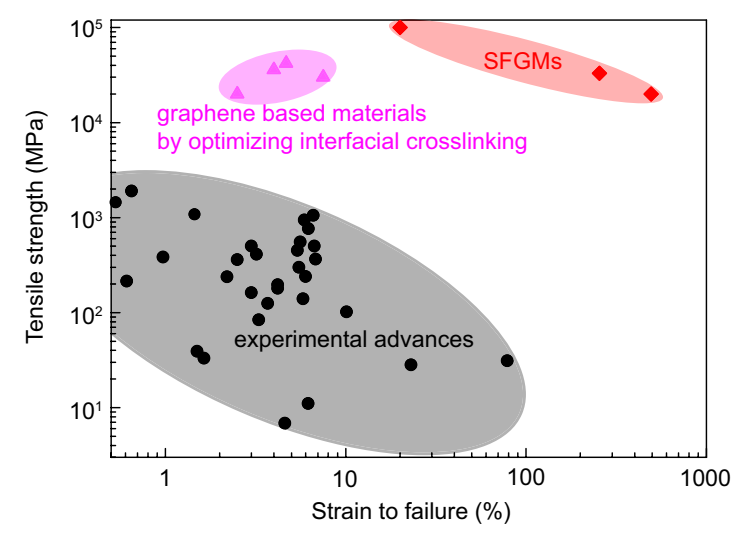

Fig. 1 Material strength-ductility paradox. Tensile strength and strain to failure of graphene-based materials reported in experimental and computational studies (Supplementary Table 1). The graphene-based materials fabricated in experiments exhibit multilevel structures and considerable defects, which are difficult to fairly compare with that from computational studies. Herein, SFGMs set a reference limit on the strength-ductility of graphene-based materials.

modifying the chemical structures of graphene building blocks. The second strategy is interfacial engineering, including hydrogen bonding, ${ }^{32}$ ionic bonding, ${ }^{33-35} \pi-\pi$ bonding, ${ }^{36-38}$ covalent bonding, $^{39-42}$ and synergistic interactions, ${ }^{17,43,44}$ which can be integrated into the graphene-based materials and thus enable the efficient load transfer among the building blocks. For example, Wan et al. ${ }^{44}$ fabricated graphene films with a tensile strength of $945 \mathrm{MPa}$ by successive application of the optimized ratio of $\pi-\pi$ bonding and covalent bonding agents. However, after decades of extensive work, some of the key mechanical performance are far lower than their ideal values; for example, the tensile strength of graphene-based materials (Supplementary Table 1) is still far lower than that of their building blocks (about $100 \mathrm{GPa}$ ). Graphenebased materials could be processed to exhibit high ductility but usually at the expense of substantial loss of strength. For example, Xiao et al. ${ }^{45}$ fabricated graphene films with rich hierarchical wrinkles and folds, which have a rubber-like mechanical behavior with remarkably large fracture strain of $23 \%$ but the tensile strength of only about $30 \mathrm{MPa}$. This strength-ductility paradox emerging in graphene-based materials (Fig. 1) largely limits their wide uses in load-bearing applications. Therefore, the rational design of the microstructures in graphene-based materials that breaks strength-ductility trade-off is highly desirable.

Evolved over billions of years, biomaterials provide a source of inspiration in designing high performance and multifunctional materials. A large number of biomaterials, for example, natural silk, feature exceptional mechanical properties including high strength and large extensibility, resulting from their folded structures of $\beta$ sheets. As shown in Fig. $2 a$, b, the $\beta$-sheet consisting of $\beta$-strands is one of the secondary elementary building blocks of protein. ${ }^{46}$ Unlike most common polymers, protein molecules consisting of one or more long chains of amino acid residues adopt specific three-dimensional conformations (called the native folds) that have a large number of favorable interactions within the protein. The self-folded structures of $\beta$-sheets (referred to conformations) and changes between them (called conformation transitions) endow the protein with fascinating mechanical properties and thus fulfill specific biological functions. ${ }^{47,48}$

In this work, inspired by the self-folded structures that endow protein with fascinating mechanical performance, we developed a self-folded topological strategy to enhance the mechanical properties of graphene-based materials by assembling graphene into self-folded graphene-based materials (SFGMs, Fig. 2c, d). The representative elementary building unit of SFGMs is similar to the simple but intriguing self-assembled graphene observed in recent experiments and simulations: the spontaneous selftearing and peeling off towards the formation of a micrometersize folded graphene. ${ }^{49-51}$ Molecular dynamics (MD) simulations of the deformation and failure of SFGMs were performed, which indicate that interfacial shearing, sliding, and unfolding among adjacent graphene interface dissipate mechanical energy and thus endow the films with exceptional strength-ductility synergy. To optimize the mechanical performance, a wide range of interface interaction and self-folded length of SFGMs were exploited. With the understanding of the underlying mechanism discussed by continuum mechanics-based model, optimal interface interaction and self-folded length of SFGMs for mechanical enhancement were identified based on our simulation results.

\section{RESULTS AND DISCUSSION}

Typical mechanical behaviors of SFGMs

The representative volume element (RVE) of SFGMs before and after tension is illustrated in Fig. 2 and Supplementary Fig. 1, where the $h_{0}, L$, and $L_{0}$ are the interlayer spacing between folded layers, total length, and self-folded length of the RVE, respectively. We first investigate the stability of SFGMs by performing simulations of SFGMs having different self-folded lengths $\left(L_{0}\right)$ and interface interactions $(\epsilon)$ under the temperature of $300 \mathrm{~K}$ for 1 ns (see Methods for details). It demonstrates that the stability of SFGMs increases with the increasing of $L_{0}$ and $\epsilon$ (Fig. 3), indicating a controlled stability by modulating the geometrical and mechanical parameters of SFGMs. Subsequently, we investigate the deformation and failure of a typical SFGM with $L_{0}$ of $38.4 \mathrm{~nm}$ and $\epsilon$ of $10 \epsilon_{0}$. The strain-stress curve is shown in Fig. 4a, where the nominal strain $(\varepsilon$, the change of length divided by the original length) and nominal stress ( $\sigma$, the tensile force divided by the original cross-sectional area) are adopted. The simulation cartoons and snapshots of SFGMs under tension are shown in Fig. $4 \mathrm{~b}$ and Supplementary Fig. 2, respectively. Herein the stretching process is divided into four stages: (I) The first stage: from point $A$ to point $B$. The stress arises rapidly with the increasing of strain until the first peak stress $\left(\sigma_{1}\right)$, named as unfolding strength. In this stage, the increasing tensile loads applied in the overall structure are transferred into the shear deformation of self-folded interface. (II) The second stage: from point $B$ to point $C$. First, there is a slight drop in stress following point $B$, and this drop is similar to that of multi-layer graphene assemblies in the study of Xia et al., ${ }^{10}$ which is attributed to the transition from static friction to dynamic friction. Subsequently, the stress changes slightly, and the average stress in the plateau regime (Fig. $4 a$ ) is named as plateau stress $\left(\sigma_{\mathrm{p}}\right)$. Afterwards, the stress decreases with the strain increasing since the interface sliding of SFGMs significantly reduces the self-folded length $L_{0}$, indicating there is a negative-stiffness region of SFGMs in this stage. The elongation of the material in the range of negative stiffness would jump if it is loaded using constant force instead of constant displacement. Despite the fact that it still dissipates mechanical energy in this range, a more rational design of SFGMs should be developed to avoid or delay the catastrophic moment of the material for practical applications. Future efforts should be directed, but not limited, at introducing multimodal and selfhealable interfaces to enable strong and tough SFGMs. ${ }^{7}$ For engineering applications, the stiffness could be modulated by introducing other microstructures, for example, interlayer crosslinks of different lengths. Additionally, the strain-rate effect observed in the simulations (Supplementary Fig. 3) can be expected to help SFGMs to damp the deformations during dynamical loading. (III) The third stage: from point $C$ to point $D$. As the strain continues to increase, the stress decreases abruptly as a 


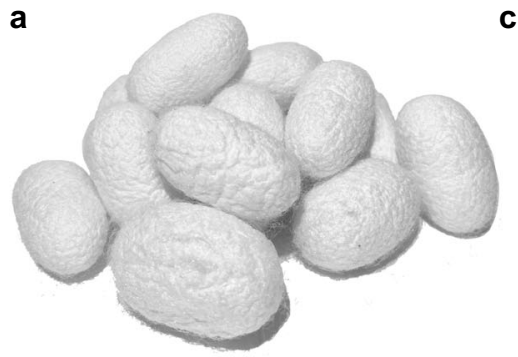

C

b

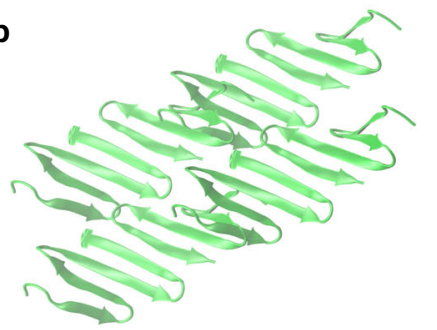

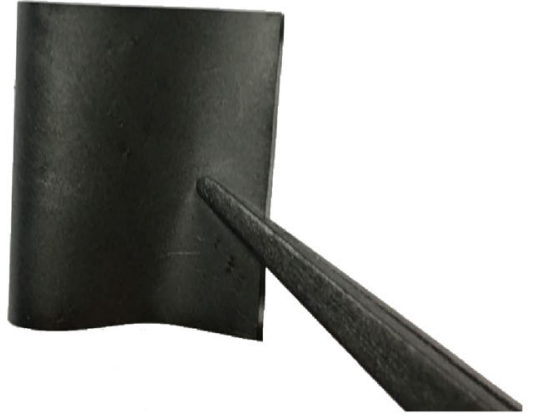

d

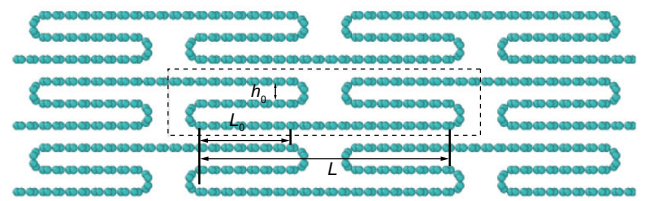

Fig. 2 Natural silk and the graphene-based materials. a Optical image of silk and $\mathbf{b}$ illustration of folded $\beta$-sheets. c Optical image of graphene-based materials and $\mathbf{d}$ illustration of SFGM structure.

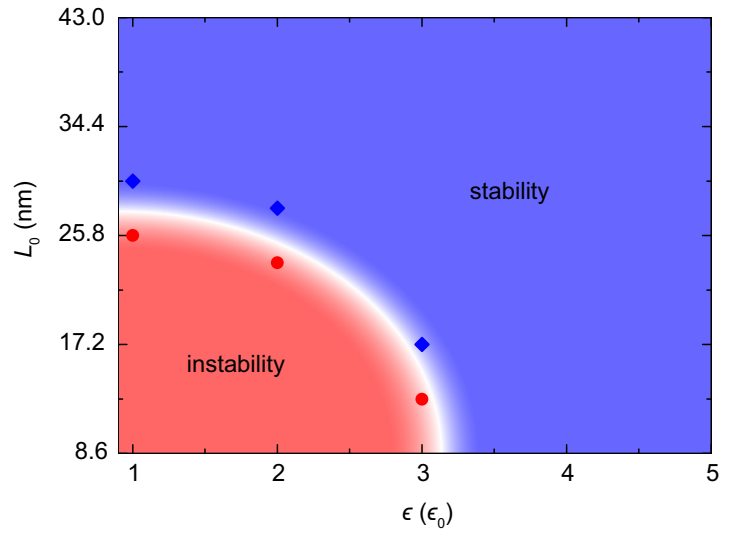

Fig. 3 Effects of the geometrical $\left(L_{0}\right)$ and mechanical $(\epsilon)$ parameters on the stability of SFGMs under the temperature of $300 \mathrm{~K}$.

critical transition from the folded multilayers to unfolded monolayer occurs. (IV) The fourth stage: from point $D$ to point E. This stage shows the mechanical behavior of completely unfolded SFGM. It is similar to that of monolayer graphene (tensile strength of about $100 \mathrm{GPa}$ and thickness of about $0.34 \mathrm{~nm}$ ), where the second peak stress (tensile strength, $\sigma_{2}$ ) is observed at the failure of this unfolded monolayer. The original thickness of SFGMs with three-folded layers is about three times of that of pristine graphene. Hence, the nominal tensile strength can be predicted as about $100 / 3=33 \mathrm{GPa}$, which is also observed in the simulation results (Fig. 4a). Additionally, the MD simulations demonstrate that the fracture strain of SFGMs could reach as high as $258 \%$.

Effect of interface interaction on the mechanical behaviors of SFGMs

To investigate the effect of different levels of interface interaction including hydrogen bonding, ${ }_{1}^{32}$ ionic bonding, ${ }^{33-35} \pi-\pi$ bonding, $^{36-38}$ covalent bonding, ${ }^{39-42}$ and synergistic interactions ${ }^{17,43,44}$ that can be integrated in experiments, simulations of SFGMs with a wide range of interface interaction were performed by modifying the Lennard-Jones potential well of AIREBO potential $(\epsilon)$. To modulate the interface interaction of SFGMs, the value of $\epsilon$ is modified as 1-30 times of the pristine value $\left(\epsilon_{0}\right)$, corresponding to the interlayer shear moduli of $0.32-10.62 \mathrm{GPa}$ benchmarked in Supplementary Fig. 4. The strain-stress curves of SFGMs with $L_{0}$ of $38.4 \mathrm{~nm}$ and $\epsilon$ from $1 \epsilon_{0}$ to $30 \epsilon_{0}$ are studied by MD simulations in Fig. $5 \mathrm{a}$, and the key mechanical properties including $\sigma_{\mathrm{p}}$ and $E_{\rho}$ (strain energy density, the area under the strain-stress curve before fracture) are summarized in Fig. 5b. Both $\sigma_{\mathrm{p}}$ and $E_{\rho}$ of SFGMs increase with the increasing of $\epsilon$ from $1 \epsilon_{0}$ to $25 \epsilon_{0}$ and then almost reach their maximum values near $\epsilon$ of $25 \epsilon_{0}$. Afterwards, $\sigma_{\mathrm{p}}$ and $E_{\rho}$ suddenly drop at $\epsilon$ of $30 \epsilon_{0}$, since the excessive interface interaction leads to the premature intralayer failure of SFGMs before sliding. Herein the interface interaction for maximizing $\sigma_{\mathrm{p}}$ and $E_{\rho}$ is defined as the optimal value. Additionally, remarked here that SFGMs with higher $\epsilon$ prefer to prematurely unfold one side because of the strain localization therein, which corresponds to the dip in stage II of the strain-stress curve (Fig. 5a, Supplementary Fig. 5).

Effect of the self-folded length on the mechanical behaviors of SFGMs

To investigate the effect of self-folded length $\left(L_{0}\right)$ on the deformation and failure of SFGMs, we conducted simulations of SFGMs with $L_{0}$ of $38.4,51.2,64.0$, and $76.8 \mathrm{~nm}$, respectively. It is found that $Y_{\mathrm{eff}}, \sigma_{1}, \sigma_{\mathrm{p}}$, and $E_{\rho}$ first increase and then converge to limited values with the increasing of $L_{0}$ (Fig. 5, Supplementary Fig. $6 \mathrm{a}, \mathrm{b})$. Since $\sigma_{1}$ closely correlates with $\sigma_{\mathrm{p}}$ and $E_{\rho}$, which determines the level of load bearing of SFGMs, the critical length of $L_{0}$ as $\sigma_{1}$ converges to a value is defined as the optimal selffolded length. We further find that the optimal self-folded length decreases with the increasing of $\epsilon$ (Supplementary Fig. 7).

As discussed previously, we have optimized the interfacial and geometrical parameters to maximize the mechanical performance of SFGMs (Fig. 5). The results show significantly enhanced mechanical properties of $\operatorname{SFGMs}\left(\sigma_{1}, \sigma_{\mathrm{p}}\right.$, and $\left.E_{\rho}\right)$ by modulating the interfacial and geometrical parameters of SFGMs ( $\epsilon$ and $\left.L_{0}\right)$. Based on these improvements, SFGMs are highly promising for uses in engineering applications. 


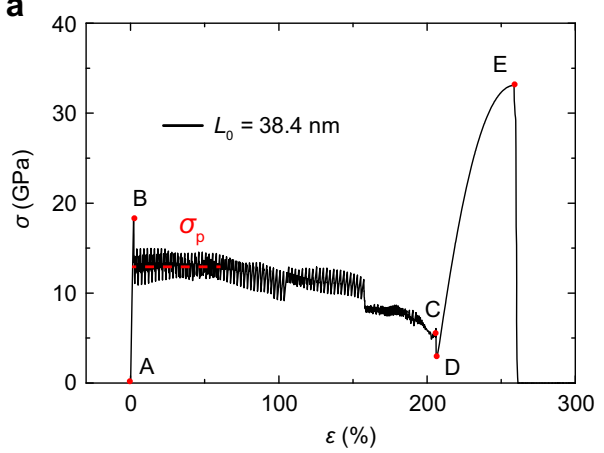

b

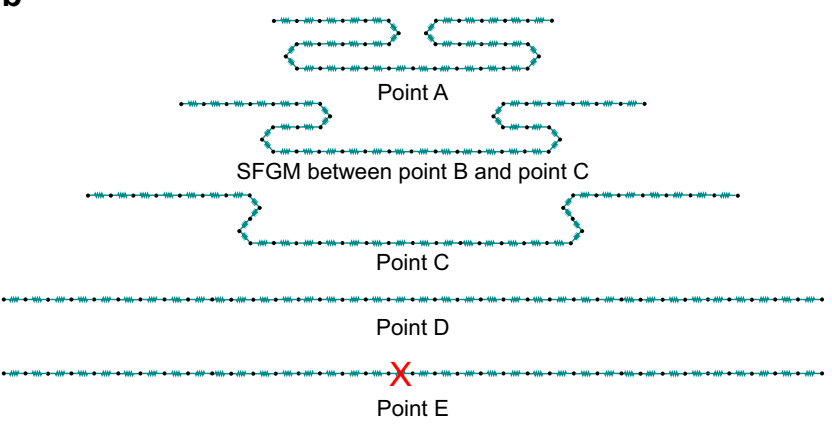

Fig. 4 Typical mechanical behaviors of SFGM. a Strain-stress curve of SFGM that can be divided into different stages. $\mathbf{b}$ Cartoons of SFGMs under tension. The snapshots of the MD simulation can be found in Supplementary Fig. 2.
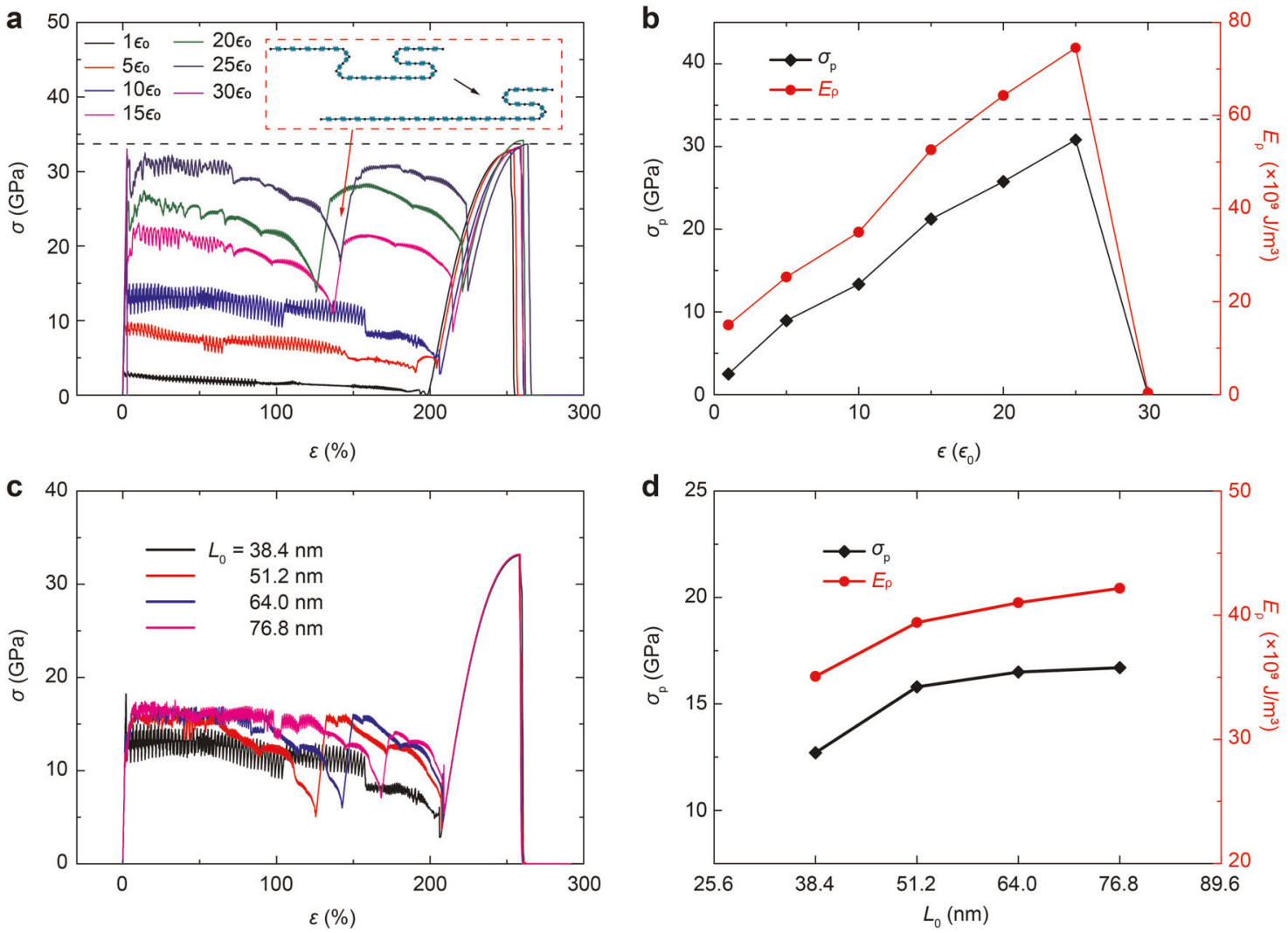

Fig. 5 Effects of mechanical and geometrical parameters on the mechanical behaviors of SFGMs. a Strain-stress curves of SFGMs having different interface interactions $(\epsilon)$, where the dashed line is about the upper limit of stress. $\mathbf{b}$ Effect of $\epsilon$ on the key mechanical properties of SFGMs. c Strain-stress curves of SFGMs having different self-folded lengths $\left(L_{0}\right)$. d Effect of $L_{0}$ on the key mechanical properties of SFGMs.

Theoretical analysis

To capture the correction between the structure and property of SFGMs, theoretical analysis is made here. As shown in Supplementary Fig. 1, the overall loads applied on SFGM are transferred into the intralayer tensile deformation and the interlayer shear deformation. Based on the deformable tension-shear (DTS) chain model, ${ }^{4}$ an effective Young's modulus $Y_{\text {eff }}$ of SFGMs can be given as

$Y_{\text {eff }}=\frac{D}{n h_{0}\left\{1 / 2+[(1+c) / s]\left(I_{0} / I_{\text {eff }}\right)\right\}}$,

where $n$ is the number of self-folded layers (Supplementary Fig. 8), $h_{0}$ is the interlayer distance between adjacent folded layers, $c$ and $s$ represent $\cosh \left(I_{\text {eff }} / I_{0}\right)$ and $\sinh \left(I_{\text {eff }} / I_{0}\right), G$ is the interlayer shear modulus, $D$ is the in-plane tensile stiffness of graphene sheets, $I_{0}=$ $\left(D h_{0} / 4 G\right)^{1 / 2}$ is the characteristic length in the model, and $l_{\text {eff }}$ is the effective self-folded length considering the difference between selffolded topology of SFGMs and the staggered arrangement of sheets in DTS model, which is assumed to linearly depend on the self-folded length ( $l_{\text {eff }}=f_{1} L_{0}$, where $f_{1}$ is a pre-factor).

In addition to $Y_{\text {eff }}$ of SFGMs, $\sigma_{1}$ closely correlates with $\sigma_{\mathrm{p}}$ and $E_{\rho \prime}$ which is greatly important to determine the level of load bearing of SFGMs. Considering the two failure mechanisms as illustrated in Supplementary Fig. 9: (1) Graphene sheets are broken before the sliding and unfolding of self-folded layers, which is denoted as 
mode G. (2) The interlayer of self-folded layers slides before the fracture of graphene sheets, which is denoted as mode I. Therefore, $\sigma_{1}$ is the lower value selected from these two failure modes, that is

$\sigma_{1}=\min \left\{\begin{array}{ll}\frac{\sigma_{\mathrm{s}}}{n} & (\text { mode } G) \\ \frac{D s \gamma_{\mathrm{cr}}}{n(1+c) l_{0}} & (\text { mode } \mathrm{I})\end{array}\right.$,

where $\sigma_{\mathrm{s}}$ is the tensile strength of monolayer graphene. In the deformation mechanism of stick-slip, the shear modulus depends linearly on the interlayer interaction $\left(G=f_{2} \epsilon\right.$, Supplementary Fig. 4). Thus $l_{\mathrm{eff}} / I_{0}$ can be given:

$\frac{l_{\text {eff }}}{I_{o}}=f_{1} L_{0} \sqrt{\frac{4 f_{2} \varepsilon}{D h_{0}}}$.

Combining Eqs. (1)-(3) and the results of MD simulations, it is found that the prediction of $\sigma_{1}$ and $Y_{\text {eff }}$ with the increasing of $L_{0}$ agrees with the simulation results (Supplementary Fig. 6a, b). Similarly, the predicted $\sigma_{1}$ and $Y_{\text {eff }}$ with the increasing of $\epsilon$ are also verified by the simulation (Supplementary Fig. $6 c$, d). It can be found that $Y_{\text {eff }}$ of SFGMs first increases and then converges to a limited value with the increasing of $L_{0}$ and $\epsilon_{0}$, and $\sigma_{1}$ of SFGMs exhibits similar evolutional trends with the increasing of $L_{0}$ and $\epsilon_{0}$.

The above-mentioned model well predicts the mechanical behaviors of SFGMs in stage I of strain-stress curve, since the linearly elastic approximation in the model is satisfied. As the strain increases, the shear-lag model fails to predict complex mechanical behaviors, such as strain localization and interlayer unfolding. Hence, we give qualitative interpretations for stages II-IV of the strain-stress curves. In stage II, the stress first experiences a slight drop because of the transition from static to dynamic friction. Then the stress reaches a plateau because the self-folded length is saturated in this region. ${ }^{8}$ Finally, the stress decreases as strain increases, resulting from the decreasing of unsaturated self-folded length after excessive interlayer sliding. For stage III, the continuum mechanics-based model does not work considering the transition from the folded multilayers to unfolded monolayer, and we cannot give its constitutive relation quantitatively. For stage IV, the SFGM completely unfolds into monolayer structure, the behavior of which is similar to that of monolayer graphene.

\section{Additional remarks}

First, the tensile strength and fracture strain of SFGMs and other graphene-based materials reported in computational and experimental studies are summarized in Fig. 1. It is remarked here that the graphene-based materials fabricated in experiments possess multi-level structures and considerable defects resulting from the fabrication process, ${ }^{52}$ which are difficult to directly compare with defect-free SFGMs. For example, a regular self-folded structure is considered in the previous discussion and the effects of defects in the building blocks and nonuniform functional groups have not been considered, which, however, are common microstructural structures of graphene-based materials and are responsible for the weakening of building blocks and load transfer. Meanwhile, interfacial engineering could enhance interface load transfer but weaken the intrinsic mechanical resistance of graphene building blocks. These dual roles of interfacial engineering discussed in our previous work ${ }^{8}$ would also exist in SFGMs if introducing interfacial engineering. In view of these facts, we expect here that the SFGMs set a reference limit on the strength-ductility of graphene-based materials and provide a guideline for the design and fabrication of graphene-based materials with high strength and ductility. With these arguments, practical SFGMs with high mechanical performance are promising to be fabricated in the future.

Second, the strength-ductility of SFGMs can be tailored by modulating the number of self-folded layers $(n)$. For example, the SFGMs with 3, 5, and 7 self-folded layers are constructed as illustrated in Supplementary Fig. 8. The original thickness of SFGMs with $n$ self-folded layers is about $n$ times of that of pristine graphene. Note that the $n$ self-folded layers would eventually unfold into one layer, and then the unfolded layer breaks down at the end of stretching SFGMs. Hence, the nominal tensile strength of SFGMs with $n$ self-folded layers can be predicted as $\sigma_{s} / n$, where $\sigma_{\mathrm{s}}$ is the tensile strength of monolayer graphene (about $100 \mathrm{GPa}$ calculated in this work). Meanwhile, the nominal fracture strain of SFGMs with $n$ self-folded layers can be approximately predicted as $\left[n\left(1+\varepsilon_{\mathrm{s}}\right) L_{0}-L_{0}\right] / L_{0}=n\left(1+\varepsilon_{\mathrm{s}}\right)-1$, where $\varepsilon_{\mathrm{s}}$ is the fracture strain of monolayer graphene (about 19\% calculated in this work). The simulation results demonstrate that the increasing of $n$ leads to the decreasing of tensile strength but increasing of fracture strain, which agrees substantially with the predictions (Fig. 6).

Finally, limited by the computational resources of full-atom simulations, we used a unit cell of SFGMs as the RVE to study the mechanical performance of SFGMs. To investigate the effect of RVE size, we conducted additional simulations of RVEs with large sizes by extending the unit cell of SFGMs in horizontal and vertical directions, respectively (Supplementary Fig. 10). The simulation results demonstrate that the extended RVEs have more folded parts, which make them possible to appear more unfolding signals of individual parts resulting from strain localization (inset of Supplementary Fig. 10a). Despite this difference, all folded parts in the RVEs of different sizes would eventually unfold into flat monolayer and thus break down, making their key mechanical
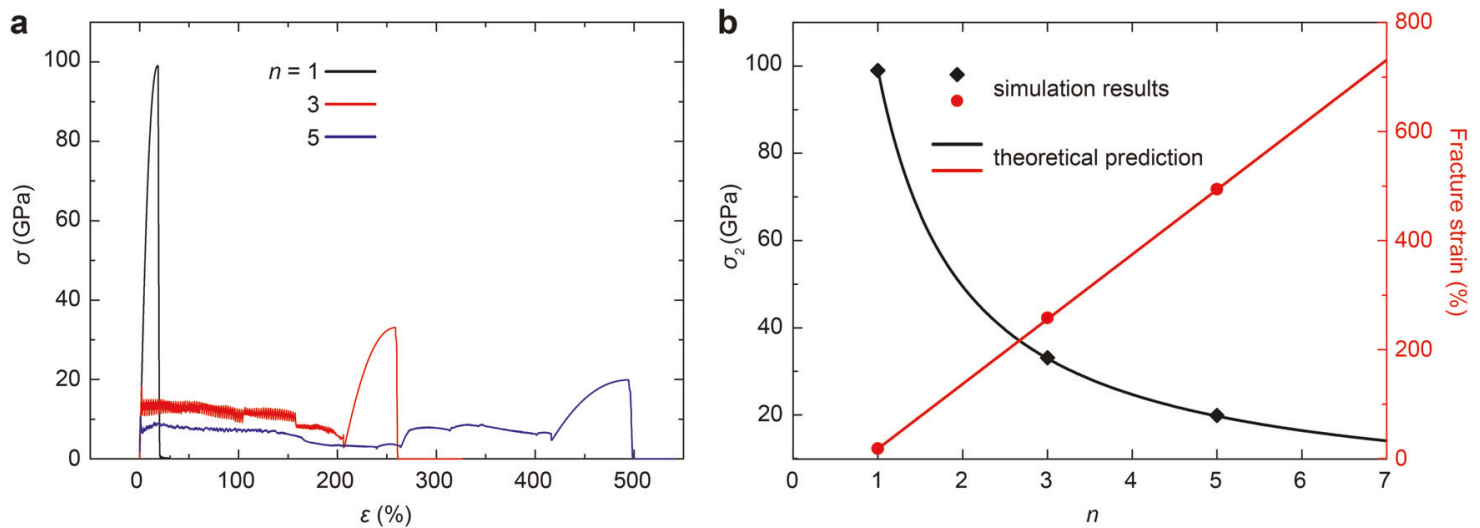

Fig. 6 Effect of the number of self-folded layers on the mechanical behaviors of SFGMs. a Stress-strain curves of SFGMs ( $n=1,3$, and 5). b Tensile strength and fracture strain of SFGMs from simulation (points) and prediction (lines). 
properties (tensile strength, fracture strain, and strain energy density) show slight difference.

To summarize, we propose a topological strategy to enhance the mechanical properties of carbon-nanoarchitected materials by self-folding graphene into macroscopic materials and investigate the overall mechanical properties of these materials having different interface interaction and self-folded length on the basis of MD simulations and continuum model-based analysis. The optimal interface interaction and self-folded length to maximize mechanical performance of SFGMs are predicted, offering a rational guide for the design and fabrication of highperformance structural materials using carbon nanostructures as building blocks. Finally, the irregular and defective microstructures neglected in our model and the room for further optimization of SFGMs are discussed, which provide a practical insight into utilizing the bio-inspired self-folding strategy in the fabrication of graphene-based materials to break the trade-off between strength and ductility.

\section{METHODS}

To explore the mechanical responses of SFGMs to tensile loads, MD simulations of uniaxial tensile tests are carried out on the RVE of SFGMs using large-scale atomic/molecular massively parallel simulator (LAMMPS) computational package. ${ }^{53}$ The adaptive intermolecular reactive empirical bond-order (AIREBO) potential ${ }^{54}$ is adopted for its abilities to capture the deformation and failure of carbon nanostructures, such as the bond breaking and formation. ${ }^{55,56}$ To avoid the original overestimation of the strength of the $\mathrm{C}-\mathrm{C}$ bonds, the potential cutoff is set to $2 \AA^{57}$ In the pristine potential, the system energy is described by

$E=\frac{1}{2} \sum_{i} \sum_{j \neq i}\left[E_{i j}^{\mathrm{REBO}}+E_{i j}^{\mathrm{LJ}}+\sum_{k \neq i, j} \sum_{l \neq i, j, k} E_{k i j l}^{\mathrm{TORS}}\right]$,

where $E^{\mathrm{REBO}}, E^{\sqcup}$ and $E^{\mathrm{TORS}}$ are the terms of covalent bonding, interface and torsion interactions, respectively. The interface interaction is determined by $E^{\llcorner J}$ with $\sqcup 12-6$ form

$V_{i j}^{\bigsqcup J}\left(r_{i j}\right)=4 \epsilon\left[\left(\frac{\sigma_{0}}{r_{i j}}\right)^{12}-\left(\frac{\sigma_{0}}{r_{i j}}\right)^{6}\right]$,

where $\sigma_{0}$ represents the distance between atoms as the potential energy is equal to 0 and $\epsilon$ defines the potential well. To investigate the effect of different level of interface interaction in SFGMs, the Lennard-Jones potential well of AIREBO potential is modified before SFGM sample is stretched to unfold accordingly. The Newton equations of motion are integrated using the Verlet algorithm with a time step of $0.5 \mathrm{fs}$. To avoid the size effect of the models, periodic boundary conditions along three dimensions are used in the simulations. Before the tensile deformation protocol is started, all constructed structures of SFGMs are fully energy minimized using a conjugate gradient algorithm. Afterwards, mechanical responses to tensile loads of these structures are investigated at $0.1 \mathrm{~K}$ using a Nosé-Hoover thermostat with a characteristic relaxation time of $0.1 \mathrm{ps}$. We also perform simulations of SFGMs under finite temperature of $300 \mathrm{~K}$ to study the effect of thermal fluctuation on the stability, deformation mechanism as well as mechanical properties of SFGMs. First, simulations of SFGMs with different $L_{0}$ and $\epsilon$ under the temperature of $300 \mathrm{~K}$ are conducted for $1 \mathrm{~ns}$, which demonstrate that the stability of SFGMs increases with increasing of $L_{0}$ and $\epsilon$ (Fig. 3). Furthermore, mechanical tests of SFGMs are conducted under the temperatures of 0.1 and $300 \mathrm{~K}$, respectively (Supplementary Fig. 11). Compared with that of SFGMs tested under the temperature of $0.1 \mathrm{~K}$, the stick-slip peaks of SFGMs during interlayer sliding process (stage II) under finite temperature of $300 \mathrm{~K}$ are reduced, which could be attributed to that the thermal fluctuation helps SFGM to overcome the energy barrier for interlayer sliding, while the tensile strength, fracture strain, and strain energy density of SFGMs exhibit slight difference, suggesting the minor effect of finite temperature of $300 \mathrm{~K}$ on the key mechanical properties of SFGMs. The uniaxial tensile strain is applied by uniaxially deforming the periodic simulation box and applying affine displacement to the atomic positions until the failure of structures, in which the other two transverse directions are equilibrated of 1 bar by coupling to a Berendsen barostat. We did a convergence test on deformation velocities, which demonstrates that the deformation velocity of $20 \mathrm{~m} / \mathrm{s}$ has a slight effect on the key mechanical performance of SFGMs (Supplementary Fig. 3). Thus, this deformation velocity is adopted, which represents a good balance between computational accuracy and efforts.

\section{DATA AVAILABILITY}

The data that support the findings of this study are available from the corresponding author upon reasonable request.

\section{CODE AVAILABILITY}

All codes of this current study are available from the corresponding author upon reasonable request.

Received: 13 July 2019; Accepted: 15 January 2020; Published online: 05 February 2020

\section{REFERENCES}

1. $\mathrm{Xu}, \mathrm{X}$. et al. Length-dependent thermal conductivity in suspended single-layer graphene. Nat. Commun. 5, 3689 (2014).

2. Castro Neto, A. H., Guinea, F., Peres, N. M. R., Novoselov, K. S. \& Geim, A. K. The electronic properties of graphene. Rev. Mod. Phys. 81, 109-162 (2009).

3. Lee, C., Wei, X., Kysar, J. W. \& Hone, J. Measurement of the elastic properties and intrinsic strength of monolayer graphene. Science 321, 385-388 (2008).

4. Liu, Y. L., Xie, B., Zhang, Z., Zheng, Q. S. \& Xu, Z. P. Mechanical properties of graphene papers. J. Mech. Phys. Solids 60, 591-605 (2012).

5. Wei, X., Naraghi, M. \& Espinosa, H. D. Optimal length scales emerging from shear load transfer in natural materials: application to carbon-based nanocomposite design. ACS Nano 6, 2333-2344 (2012).

6. He, Z., Zhu, Y., Xia, J. \& Wu, H. Optimization design on simultaneously strengthening and toughening graphene-based nacre-like materials through noncovalent interaction. J. Mech. Phys. Solids 133, 103706 (2019).

7. Liu, Y. L. \& Xu, Z. P. Multimodal and self-healable interfaces enable strong and tough graphene-derived materials. J. Mech. Phys. Solids 70, 30-41 (2014).

8. Gao, E., Cao, Y., Liu, Y. \& Xu, Z. Optimizing interfacial cross-linking in graphenederived materials, which balances intralayer and interlayer load transfer. ACS Appl. Mater. Interfaces 9, 24830-24839 (2017).

9. Ruiz, L., Xia, W. J., Meng, Z. X. \& Keten, S. A coarse-grained model for the mechanical behavior of multi-layer graphene. Carbon 82, 103-115 (2015).

10. Xia, W., Ruiz, L., Pugno, N. M. \& Keten, S. Critical length scales and strain localization govern the mechanical performance of multi-layer graphene assemblies. Nanoscale 8, 6456-6462 (2016).

11. Xia, W. J., Song, J. K., Meng, Z. X., Shao, C. \& Keten, S. Designing multi-layer graphene-based assemblies for enhanced toughness in nacre-inspired nanocomposites. Mol. Syst. Des. Eng. 1, 40-47 (2016).

12. Meng, Z. X. et al. Spalling-like failure by cylindrical projectiles deteriorates the ballistic performance of multi-layer graphene plates. Carbon 126, 611-619 (2018).

13. Wei, X. et al. Recoverable slippage mechanism in multilayer graphene leads to repeatable energy dissipation. ACS Nano 10, 1820-1828 (2016).

14. Li, P. et al. In situ tensile fracturing of multilayer graphene nanosheets for their inplane mechanical properties. Nanotechnology 30, 475708 (2019).

15. Lee, J. H., Loya, P. E., Lou, J. \& Thomas, E. L. Dynamic mechanical behavior of multilayer graphene via supersonic projectile penetration. Science 346, 1092-1096 (2014).

16. Meyer, J. C. et al. Direct imaging of lattice atoms and topological defects in graphene membranes. Nano Lett. 8, 3582-3586 (2008).

17. Wan, S., Fang, S., Jiang, L., Cheng, Q. \& Baughman, R. H. Strong, conductive, foldable graphene sheets by sequential ionic and $\pi$ bridging. Adv. Mater. 30, e1802733 (2018).

18. Zheng, Q. et al. Transparent conductive films consisting of ultralarge graphene sheets produced by Langmuir-Blodgett assembly. ACS Nano 5, 6039-6051 (2011).

19. Zhao, J., Pei, S., Ren, W., Gao, L. \& Cheng, H. M. Efficient preparation of large-area graphene oxide sheets for transparent conductive films. ACS Nano 4, 5245-5252 (2010).

20. Xu, Z., Sun, H., Zhao, X. \& Gao, C. Ultrastrong fibers assembled from giant graphene oxide sheets. Adv. Mater. 25, 188-193 (2013).

21. Xin, G. et al. Highly thermally conductive and mechanically strong graphene fibers. Science 349, 1083-1087 (2015).

22. Peng, L. et al. Ultrahigh thermal conductive yet superflexible graphene films. Adv. Mater. 29, 1700589 (2017). 
23. Lin, X. et al. Fabrication of highly-aligned, conductive, and strong graphene papers using ultralarge graphene oxide sheets. ACS Nano 6, 10708-10719 (2012).

24. Kumar, P. et al. Large-area reduced graphene oxide thin film with excellent thermal conductivity and electromagnetic interference shielding effectiveness. Carbon 94, 494-500 (2015).

25. Chen, J. et al. Size fractionation of graphene oxide sheets via filtration through track-etched membranes. Adv. Mater. 27, 3654-3660 (2015).

26. Xin, G. et al. Large-area freestanding graphene paper for superior thermal management. Adv. Mater. 26, 4521-4526 (2014).

27. Shen, B., Zhai, W. T. \& Zheng, W. G. Ultrathin flexible graphene film: an excellent thermal conducting material with efficient EMI shielding. Adv. Funct. Mater. 24, 4542-4548 (2014).

28. Chen, H., Muller, M. B., Gilmore, K. J., Wallace, G. G. \& Li, D. Mechanically strong, electrically conductive, and biocompatible graphene paper. Adv. Mater. 20, 3557-3561 (2008).

29. Becerril, H. A. et al. Evaluation of solution-processed reduced graphene oxide films as transparent conductors. ACS Nano 2, 463-470 (2008).

30. Zhang, $M$. et al. Multifunctional pristine chemically modified graphene films as strong as stainless steel. Adv. Mater. 27, 6708-6713 (2015).

31. Kumar, P. V. et al. Scalable enhancement of graphene oxide properties by thermally driven phase transformation. Nat. Chem. 6, 151-158 (2014).

32. Medhekar, N. V., Ramasubramaniam, A., Ruoff, R. S. \& Shenoy, V. B. Hydrogen bond networks in graphene oxide composite paper: structure and mechanical properties. ACS Nano 4, 2300-2306 (2010).

33. Park, S. et al. Graphene oxide papers modified by divalent ions-enhancing mechanical properties via chemical cross-linking. ACS Nano 2 572-578 (2008).

34. Lam, Do. V. et al. A robust and conductive metal-impregnated graphene oxide membrane selectively separating organic vapors. Chem. Commun. 51, 2671-2674 (2015).

35. Yeh, C. N., Raidongia, K., Shao, J., Yang, Q. H. \& Huang, J. On the origin of the stability of graphene oxide membranes in water. Nat. Chem. 7, 166-170 (2014).

36. Xu, Y., Bai, H., Lu, G., Li, C. \& Shi, G. Flexible graphene films via the filtration of water-soluble noncovalent functionalized graphene sheets. J. Am. Chem. Soc. 130, 5856-5857 (2008)

37. Liu, Y. et al. Giant enhancement in vertical conductivity of stacked CVD graphene sheets by self-assembled molecular layers. Nat. Commun. 5, 5461 (2014).

38. Wan, S. et al. Ultrastrong graphene films via long-chain $\pi$-bridging. Matter $\mathbf{1}$, 389-401 (2019)

39. Gao, Y. et al. The effect of interlayer adhesion on the mechanical behaviors of macroscopic graphene oxide papers. ACS Nano 5, 2134-2141 (2011).

40. Park, S., Dikin, D. A., Nguyen, S. T. \& Ruoff, R. S. Graphene oxide sheets chemically cross-linked by polyallylamine. J. Phys. Chem. C. 113, 15801-15804 (2009).

41. Tian, Y., Cao, Y., Wang, Y., Yang, W. \& Feng, J. Realizing ultrahigh modulus and high strength of macroscopic graphene oxide papers through crosslinking of mussel-inspired polymers. Adv. Mater. 25, 2980-2983 (2013).

42. Kim, Y. S. et al. Easy preparation of readily self-assembled high-performance graphene oxide fibers. Chem. Mater. 26, 5549-5555 (2014).

43. Wan, S. et al. Use of synergistic interactions to fabricate strong, tough, and conductive artificial nacre based on graphene oxide and chitosan. ACS Nano 9 9830-9836 (2015).

44. Wan, S. et al. Sequentially bridged graphene sheets with high strength, toughness, and electrical conductivity. Proc. Natl Acad. Sci. USA 115, 5359-5364 (2018).

45. Xiao, Y. et al. Sheet collapsing approach for rubber-like graphene papers. ACS Nano 11, 8092-8102 (2017).

46. Keten, S., Xu, Z., Ihle, B. \& Buehler, M. J. Nanoconfinement controls stiffness, strength and mechanical toughness of $\beta$-sheet crystals in silk. Nat. Mater. 9 359-367 (2010)

47. Carlsson, U. \& Jonsson, B.-H. Folding of $\beta$-sheet proteins. Curr. Opin. Struct. Biol. 5 , 482-487 (1995).

48. Jager, $M$. et al. Understanding the mechanism of $\beta$-sheet folding from a chemical and biological perspective. Biopolymers 90, 751-758 (2008).

49. Annett, J. \& Cross, G. L. Self-assembly of graphene ribbons by spontaneous selftearing and peeling from a substrate. Nature 535, 271-275 (2016).

50. Fonseca, A. F. \& Galvao, D. S. Self-tearing and self-peeling of folded graphene nanoribbons. Carbon 143, 230-239 (2019).
51. He, Z. Z., Zhu, Y. B. \& Wu, H. A. Self-folding mechanics of graphene tearing and peeling from a substrate. Front. Phys. 13, 138111 (2018).

52. Wen, Y. et al. Chemically modified graphene films with tunable negative Poisson's ratios. Nat. Commun. 10, 2446 (2019).

53. Plimpton, S. Fast parallel algorithms for short-range molecular-dynamics. J. Comput. Phys. 117, 1-19 (1995).

54. Brenner, D. W. et al. A second-generation reactive empirical bond order (REBO) potential energy expression for hydrocarbons. J. Phys. Condens. Matter 14, 783-802 (2002)

55. Wei, C. Y., Srivastava, D. \& Cho, K. J. Thermal expansion and diffusion coefficients of carbon nanotube-polymer composites. Nano Lett. 2, 647-650 (2002).

56. Song, Z., Artyukhov, V. I., Wu, J., Yakobson, B. I. \& Xu, Z. Defect-detriment to graphene strength is concealed by local probe: the topological and geometrical effects. ACS Nano 9, 401-408 (2015).

57. MaN, Dewapriya \& Meguid, S. A. Tailoring fracture strength of graphene. Comput. Mater. Sci. 141, 114-121 (2018).

\section{ACKNOWLEDGEMENTS}

This work was supported by the National Natural Science Foundation of China (11902225, 11632009, 11872284, and 11602175), the "Thousand Youth Talents Plan" from China, the Natural Science Foundation of Hubei Province (2019CFB174), the Fundamental Research Funds for the Central Universities, and the Large-scale Instrument and Equipment Sharing Foundation of Wuhan University. The numerical calculations in this work have been done on the supercomputing system in the Supercomputing Center of Wuhan University.

\section{AUTHOR CONTRIBUTIONS}

E.G. conceived the idea. Z.L. provided advices on this work. X.J. and E.G. carried out the simulation and related data analysis. All authors participated in the writing of the manuscript.

\section{COMPETING INTERESTS}

The authors declare no competing interests.

\section{ADDITIONAL INFORMATION}

Supplementary information is available for this paper at https://doi.org/10.1038/ s41524-020-0279-8.

Correspondence and requests for materials should be addressed to E.G.

Reprints and permission information is available at http://www.nature.com/ reprints

Publisher's note Springer Nature remains neutral with regard to jurisdictional claims in published maps and institutional affiliations.

Open Access This article is licensed under a Creative Commons Attribution 4.0 International License, which permits use, sharing, adaptation, distribution and reproduction in any medium or format, as long as you give appropriate credit to the original author(s) and the source, provide a link to the Creative Commons license, and indicate if changes were made. The images or other third party material in this article are included in the article's Creative Commons license, unless indicated otherwise in a credit line to the material. If material is not included in the article's Creative Commons license and your intended use is not permitted by statutory regulation or exceeds the permitted use, you will need to obtain permission directly from the copyright holder. To view a copy of this license, visit http://creativecommons. org/licenses/by/4.0/.

(c) The Author(s) 2020 